\title{
Diagnostica metabolica della calcolosi renale. Casi clinici (Parte II)
}

\author{
Corrado Vitale ${ }^{1}$, Amelia Rodofili ${ }^{1}$, Francesca Bermond ${ }^{1}$, Alberto Tricerri ${ }^{1}$, Martino Marangella ${ }^{2}$ \\ ${ }^{1}$ SC Nefrologia e Dialisi, AO Ordine Mauriziano di Torino, Torino \\ ${ }^{2}$ Fondazione Scientifica Mauriziana ONLUS, Torino
}

\begin{abstract}
Metabolic diagnosis of nephrolithiasis. Clinical cases (Part II)
This is the second part of an article on the metabolic diagnosis of nephrolithiasis, the first part of which was published in the previous issue of this journal. Here we report on three clinical cases representative of a rational diagnostic workup of nephrolithiasis in specific clinical contexts: secondary hyperuricemia, cystinuria, and primary hyperoxaluria. Nephrolithiasis is one of the most frequent causes of hospitalization in nephrology and urology units in our country and is an important source of discomfort in affected patients. Despite the availability of modern, minimally invasive endourological procedures to remove stones from the urinary tract, prevention of stone recurrences remains an essential strategy. Chemical analysis of stones, whether passed spontaneously or removed surgically, is a crucial step in etiological diagnostics aimed at devising adequate prevention strategies. Modern endoscopic lithotripsy techniques provide complete fragmentation of stones and, compared to extracorporeal shock wave lithotripsy, have the advantage of avoiding painful elimination of fragments through the urinary tract. This means, however, that stone fragments are often unavailable for analysis, thereby depriving the nephrologist of an important tool for diagnosis. As a consequence, metabolic evaluation tends to be the only means of establishing the etiology of stones and preventing their recurrence. We conclude that close collaboration between nephrologists and urologists is a prerequisite for optimizing the diagnosis and treatment of stone disease.
\end{abstract}

Keywords: Cystinuria, Hyperoxaluria, Nephrolithiasis, Primary hyperparathyroidism, Urolithiasis

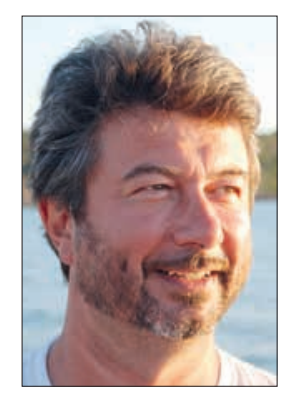

Corrado Vitale

\section{Caso Clinico 3}

Anamnesi

Nata nel 1995, studentessa, in followup dalla nascita presso Centro Ematologico pediatrico per iper-leucocitosi idiopatica (GB 20.000), giunta alla nostra osservazione per riscontro di nefrolitiasi all'età di 15 anni.

Dall'età di 12 anni, la paziente assumeva allopurinolo $300 \mathrm{mg} /$ die per una marcata iperuricemia, verosimilmente ricon-

Accepted: September 26, 2016

Published online: November 9, 2016

Indirizzo per la corrispondenza:

Corrado Vitale

SC di Nefrologia e Dialisi

AO Ordine Mauriziano

Largo Turati 62

10128 Torino

covitale@mauriziano.it ducibile al disordine emoproliferativo. La sua alimentazione era piuttosto disordinata e l'assunzione idrica raramente superava il litro al giorno. Non aveva mai lamentato disturbi urinari.

Nell'agosto del 2010 fu ricoverata in Pronto Soccorso per colica renale sinistra, senza espulsione di calcoli. All'ecografia renale furono riscontrati un calcolo del diametro massimo di $35 \mathrm{~mm}$ localizzato nella pelvi renale destra e un calcolo di $14 \mathrm{~mm}$ in un calice inferiore del rene destro. II dato fu, in seguito, confermato mediante TAC senza mdc.

Risolta la sintomatologia acuta, la paziente fu dimessa dal Pronto Soccorso con prescrizione di proseguire con terapia idropinica e allopurinolo e l'indicazione di sottoporsi a visita urologica per pianificare la bonifica chirurgica della nefrolitiasi.

Il medico di Medicina Generale inviò la paziente anche a visita nefrologica presso il nostro Centro, dove ella giunse in buone condizioni generali, clinicamente asintomatica e già in lista d'attesa per l'intervento urologico.

\section{Indagini biochimiche e strumentali}

Trattandosi di una litiasi in un soggetto giovane con un'importante comorbilità, fu prescritto uno screening metabolico completo (Fig. 1). 
Nel percorso diagnostico della nefrolitiasi, un'attendibile determinazione della composizione dei calcoli rappresenta un momento fondamentale (1). In questo caso, stante l'indisponibilità di frammenti litiasici, come primo orientamento fu prescritto un radiogramma diretto dell'apparato urinario, informativo sul grado di radio-opacità dei calcoli (intensa negli uroliti contenenti calcio, debole o del tutto assente in quelli costituiti da acido urico). Per questo scopo, il radiogramma diretto è spesso più indicativo rispetto alla TAC standard, dove tanto la litiasi urica quanto quella calcica appaiono radio-opache. Benché sia possibile impostare una diagnostica differenziale utilizzando le Unità Hounsfield, questo metodo

\footnotetext{
Siero: urea, creatinina, acido urico, sodio, potassio, calcio totale e ionizzato, fosforo, cloro, magnesio, $\mathrm{pH}$, bicarbonato, PTH, $25(\mathrm{OH})$ Vitamina $\mathrm{D}_{3}$, fosfatasi alcalina.

Urine delle 24 ore: urea, creatinina, acido urico, sodio, potassio, calcio, fosforo, cloro, magnesio, ossalato, citrato, solfato, $\mathrm{pH}$, bicarbonato, ammonio, acidità titolabile. Test di Brand.
}

Urine a digiuno: calcio, citrato, creatinina, desossipiridinolina.

Fig. 1 - Screening metabolico per calcolosi renale. Indagini bioumorali sieriche e urinarie. non è ancora sufficientemente preciso per consentire di definire mediante la TAC la natura chimica dei calcoli renali (2).

Al radiogramma diretto dell'apparato urinario fu riscontrata un'immagine del diametro massimo di circa $30 \mathrm{~mm}$, di tenuissima radio-opacità, proiettantesi in corrispondenza della pelvi renale sinistra, mentre non risultarono evidenti immagini radio-opache né in corrispondenza dell'ombra renale destra né lungo il presumibile decorso delle vie escretrici urinarie.

I risultati più significativi delle prime indagini di laboratorio $(10 / 2010)$ e di quelle eseguite successivamente sono riportati nella Tabella I. Abitualmente, il primo controllo metabolico viene effettuato in condizioni basali, a dieta libera e senza terapia farmacologica. Nel caso di questa paziente, considerando che l'allopurinolo era stato prescritto anni prima per una severa iperuricemia e che la litogenesi urinaria doveva necessariamente essersi sviluppata nel frattempo, non si ritenne opportuno sospendere la terapia.

\section{Diagnosi}

Al controllo del 10/2010 (Tab. I), i livelli di acido urico sierici e urinari apparvero francamente elevati, soprattutto considerando che la paziente era già in terapia con $300 \mathrm{mg} /$ die di allopurinolo.

I principali effettori condizionanti elevati valori di BAU sono l'iperuricuria, l'iperacidità urinaria e lo scarso volume

TABELLA I - Caso clinico 3. Dati metabolici

\begin{tabular}{|c|c|c|c|c|c|}
\hline & Val. normali & $10 / 2010$ & $10 / 2011$ & $2 / 2013$ & $5 / 2015$ \\
\hline Creatininemia (mg/dL) & $0.5-1.3$ & 0.6 & 0.6 & 0.6 & 0.7 \\
\hline Clear. Creatinina $(\mathrm{mL} / \mathrm{min})$ & $70-140$ & 99 & 95 & 101 & 86 \\
\hline Calcemia totale $(\mathrm{mg} / \mathrm{dL})$ & $8.2-10.2$ & 9.7 & 9.6 & 9.5 & 8.9 \\
\hline $\mathrm{Ca}++$ sierico $(\mathrm{mmol} / \mathrm{L})$ & $1.15-1.30$ & 1.24 & 1.21 & 1.22 & 1.23 \\
\hline Fosforemia (mg/dL) & $2.5-4.5$ & 4.0 & 3.9 & 3.8 & 4 \\
\hline PTH intatto $(p g / m L)$ & $11-70$ & 36 & - & - & - \\
\hline Uricemia (mg/dL) & $3.4-7$ & 7.3 & 7.1 & 6.6 & 6.6 \\
\hline Fosfaturia $(\mathrm{mmol} / 24 \mathrm{~h})$ & $13-52$ & 18.6 & - & - & 24.8 \\
\hline Calciuria (mmol/24h) & $2.5-9$ & 2.5 & 2.7 & - & 3 \\
\hline Calciuria (mg/kg) & $<4$ & 2.2 & 2.3 & - & 2.1 \\
\hline Ossaluria (mmol/24h) & $<0.55$ & 0.25 & 0.28 & - & 0.41 \\
\hline Citraturia (mmol/24h) & $1.5-6.5$ & 1.8 & 2.3 & - & 1.7 \\
\hline Uricuria (mg/24h) & $350-900$ & 868 & 513 & 570 & 1246 \\
\hline $\mathrm{pH}$ urinario & $5-7$ & 5.59 & 6.38 & 5.40 & 6.1 \\
\hline Diuresi $(m L / 24 h)$ & & 1130 & 2650 & 840 & 1660 \\
\hline B $\mathrm{CaOx}$ & Satur. $=1$ & 4.7 & 1.7 & - & \\
\hline$B A U$ & Satur. $=1$ & 2.1 & 0.15 & 2.7 & 0.9 \\
\hline B bsh & Satur. $=1$ & 0.7 & 0.65 & - & \\
\hline
\end{tabular}


urinario $(3,4)$. In questo caso, tutti questi fattori di rischio coesistevano e la ßAU risultava decisamente elevata.

Considerando anche la debole o nulla radio-opacità dei calcoli renali evidenziati all'ecografia, fu posto il sospetto diagnostico di litiasi urica.

\section{Terapia}

Le dimensioni dei calcoli renali (in particolare di quello pielico destro di $30 \mathrm{~mm}$ ) avevano motivato l'avvio della paziente a un programma di rimozione chirurgica.

Nella nostra esperienza è tuttora piuttosto frequente che, a seguito del riscontro mediante TAC di una litiasi renale di dimensioni tali da impedire l'espulsione spontanea, il paziente sia avviato a intervento chirurgico anche senza una valutazione metabolica preliminare. Quest'ultima può essere, invece, molto utile, giacché, nel caso della litiasi urica, la chemiolisi ha concrete possibilità di successo, permettendo di evitare (quando non vi sia ostruzione acuta delle vie urinarie) il ricorso a terapie invasive.

In questo caso, il volume dei calcoli era decisamente consistente. Tuttavia, non vi era alcuna evidenza di uropatia ostruttiva e la paziente era riluttante a sottoporsi a un intervento chirurgico. Fu, dunque, proposto un tentativo di litolisi farmacologica, prescrivendo una terapia idropinica spinta, aumentando la posologia dell'allopurinolo a $300 \mathrm{mg} \times 2$ e alcalinizzando le urine con citrato di potassio ( 2 grammi 3 volte al giorno) (5).

\section{Decorso clinico}

La paziente fu rivalutata a un anno di distanza (Tab. I, ottobre 2011). Grazie a una discreta riduzione dell'uricuria e a un congruo aumento della diuresi e del $\mathrm{pH}$ urinario, le urine risultarono marcatamente sottosature rispetto all'acido urico, come indicato da valori di ßAU inferiori di oltre 10 volte rispetto ai precedenti. Il peculiare andamento della sovrasaturazione urinaria per l'acido urico in funzione dei valori di $\mathrm{pH}$ urinario è illustrato nella Figura 2.

Nello stesso periodo, un controllo ecografico riscontrò "... reni di forma e dimensioni regolari, con rapporti corticomidollari conservati. Minima ectasia delle vie escretrici a sinistra, pelvi normale a destra. Un calcolo di 6-7 $\mathrm{mm}$ in un calice inferiore del rene destro e due calcoli di 4-5 $\mathrm{mm}$ nei gruppi caliceali medi e inferiori di sinistra". Dopo un anno di terapia mirata, la volumetria dei calcoli risultò, dunque, estremamente ridotta, motivando la scelta di proseguire con la sola terapia medica.

Due mesi dopo (dicembre 2011), la paziente riferì l'espulsione di alcuni minuti frammenti litiasici, purtroppo dispersi e non analizzati.

Un'ecografia del gennaio del 2012 documentò la completa assenza di calcoli bilateralmente.

Al controllo del febbraio del 2013, la paziente presentò

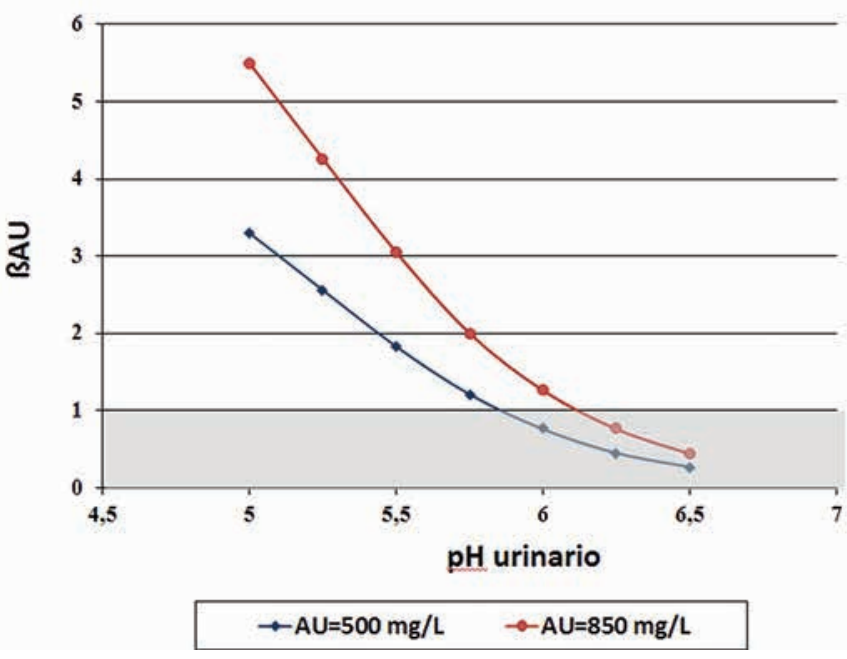

Fig. 2 - Effetti della variazione del pH sullo stato di saturazione urinaria per l'acido urico ( $(\mathrm{AU})$ ), calcolati per due differenti valori di uricuria.

un quadro laboratoristico francamente peggiorato rispetto ai precedenti, con una marcata sovrasaturazione per l'acido urico sostenuta essenzialmente da un ridotto volume di urine marcatamente acide, espressione di scarsa aderenza terapeutica (Tab. I). L'ecografia renale documentò la comparsa di due nuovi piccoli calcoli renali a destra, poi espulsi spontaneamente nei mesi successivi e risultati, all'analisi spettrofotometrica, composti da acido urico.

Ripristinata correttamente la terapia farmacologica, con controllo ecografico nel maggio del 2014, la paziente appariva nuovamente libera da recidive di litiasi.

Nel maggio del 2015, in corso di terapia farmacologica immodificata, fu osservato un picco di iperuricuria, probabile espressione di iperproduzione di acido urico da parte di tessuto emopoietico iperplastico (Tab. I). Era, infatti, presente una cospicua splenomegalia, con diametro splenico di $25 \mathrm{~cm}$, poi trattata con splenectomia.

Al controllo del dicembre del 2015, la paziente risultò ancora libera da recidive litiasiche.

\section{Conclusioni}

Nei casi in cui le dimensioni dei calcoli renali suggeriscano l'opportunità di una rimozione chirurgica e laddove non sussistano indicazioni di urgenza, è sempre consigliabile eseguire una valutazione metabolica preliminare, al fine di identificare i casi potenzialmente suscettibili di terapia medica.

La semplice dimostrazione di calcoli scarsamente radioopachi (se non del tutto radiotrasparenti) al radiogramma diretto addominale è fortemente suggestiva di litiasi urica.

In una consistente percentuale di casi, la litiasi urica è indotta da iperacidità urinaria e scarsa diuresi, senza evidenza di iperuricuria $(3,6)$. 
In questi casi, dove si intenda perseguire la dissoluzione dei calcoli (che può richiedere tempi lunghi), è consigliabile che alla terapia alcalinizzante urinaria, volta a ottenere valori di $\mathrm{pH}$ tra 6.5 e 7, venga associato comunque l'allopurinolo a dosi piene, al fine di ridurre quanto possibile la sovrasaturazione urinaria per l'acido urico.

Ottenuta la detersione completa dei calcoli, laddove non sussista iperuricuria, la sola terapia idropinica e alcalinizzante urinaria può essere sufficiente a sottosaturare le urine e a prevenire le recidive.

\section{Caso clinico n 4}

\section{Anamnesi}

Paziente nata nel 1967, giunta a visita nefrologica per la prima volta all'età di 47 anni per riscontro occasionale di nefrolitiasi. In anamnesi: due gravidanze regolari e ovariectomia sinistra per cisti a 39 anni. Incipiente menopausa. Mai coliche renali.

La paziente riferiva di avere un fratello affetto da calcolosi renale severa sin dall'età adolescenziale, sottoposto a numerosi interventi chirurgici di litotomia (open surgery, PUL, ESWL, litotrissia endoscopica), andato incontro a insufficienza renale da pielonefrite cronica bilaterale e, dall'età di 56 anni, in terapia dialitica trisettimanale.

Al momento della visita, la paziente non aveva informazioni in merito alla natura della calcolosi del fratello. Peraltro, colpita dal suo grave decorso clinico, fin dall'età adolescenziale ella fu indotta a ingerire molti liquidi (circa 3 litri di acqua al giorno) e, a imitazione del fratello, ad assumere un cucchiaino di bicarbonato di sodio al giorno.

Nel 2014, dal momento che la paziente lamentava lombalgie persistenti, il Medico di Medicina Generale prescrisse un'ecografia addominale, nel corso della quale furono riscontrati "... a sinistra, un calcolo mesorenale di $5 \mathrm{~mm}$; a destra, un calcolo polare inferiore di $6 \mathrm{~mm}^{\prime \prime}$ e un Radiogramma diretto dell'addome che, nei limiti imposti da una mediocre toeletta intestinale, non rilevò segni certi di urolitiasi radio-opaca. Di
TABELLA II - Caso clinico 4. Dati metabolici

\begin{tabular}{lcc}
\hline & Val. normali & \\
\hline Creatininemia $(\mathrm{mg} / \mathrm{dL})$ & $0.5-1.3$ & 0.9 \\
Clear. Creatinina $(\mathrm{mL} / \mathrm{min})$ & $70-140$ & 76 \\
Uricemia $(\mathrm{mg} / \mathrm{dL})$ & $3.4-7$ & 4.4 \\
Calcemia totale $(\mathrm{mg} / \mathrm{dL})$ & $8.2-10.2$ & 8.8 \\
Fosforemia $(\mathrm{mg} / \mathrm{dL})$ & $2.5-4.5$ & 3.1 \\
Fosfaturia $(\mathrm{mmol} / 24 \mathrm{~h})$ & $13-52$ & 23.2 \\
Calciuria $(\mathrm{mmol} / 24 h)$ & $2.5-9$ & 3.1 \\
Calciuria $(\mathrm{mg} / \mathrm{kg})$ & $<4$ & 2.1 \\
Ossaluria $(\mathrm{mmol} / 24 h)$ & $<0.55$ & 0.31 \\
Citraturia $(\mathrm{mmol} / 24 h)$ & $1.5-6.5$ & 3.8 \\
Uricuria $(\mathrm{mg} / 24 h)$ & $250-900$ & 364 \\
pH urinario & $5-7$ & 6.5 \\
Diuresi $(\mathrm{mL} / 24 \mathrm{~h})$ & & 3100 \\
B CaOx & Satur. $=1$ & 2.8 \\
B bsh & Satur. $=1$ & 1.1 \\
B AU & Satur. $=1$ & 0.07 \\
Test di Brand & Negativo & Positivo \\
\hline
\end{tabular}

sua iniziativa, il Curante completò gli accertamenti con un panel di indagini metaboliche e inviò la paziente alla nostra osservazione.

\section{Indagini biochimiche e strumentali}

La diagnostica morfologica, radiologica ed ecografica risultò suggestiva per un tipo di nefrolitiasi apparentemente radiotrasparente o assai debolmente radio-opaca, la più frequente delle quali è la calcolosi urica.

TABELLA III - Caso clinico 4. Escrezione urinaria di Cistina, basale e in corso di terapia con Tiopronina

\begin{tabular}{lccc}
\hline & Val. normali & Basale & Tiopronina \\
\hline Diuresi $(\mathrm{mL} / 24 \mathrm{~h})$ & & 3640 & 3700 \\
$\mathrm{pH}$ urine & $5-7$ & 6.5 & 3.28 \\
Cistina totale urinaria $(\mu \mathrm{mol} / 24 \mathrm{~h})$ & $43-117$ & 3687 & 1013 \\
Cistina totale urinaria $(\mu \mathrm{mol} / \mathrm{L})$ & $5-22$ & 681 & 932 \\
Cistina/Creatinina $(\mu \mathrm{mol} / \mathrm{mmol})$ & & 0 & 624 \\
Tiopronina totale urinaria $(\mu \mathrm{mol} / 24 \mathrm{~h})$ & & 0 & 3207 \\
Tiopronina totale urinaria $(\mu \mathrm{mol} / \mathrm{L})$ & & 1013 & 867 \\
Cistina libera urinaria $(\mu \mathrm{mol} / \mathrm{L})$ & & 499 \\
\hline
\end{tabular}




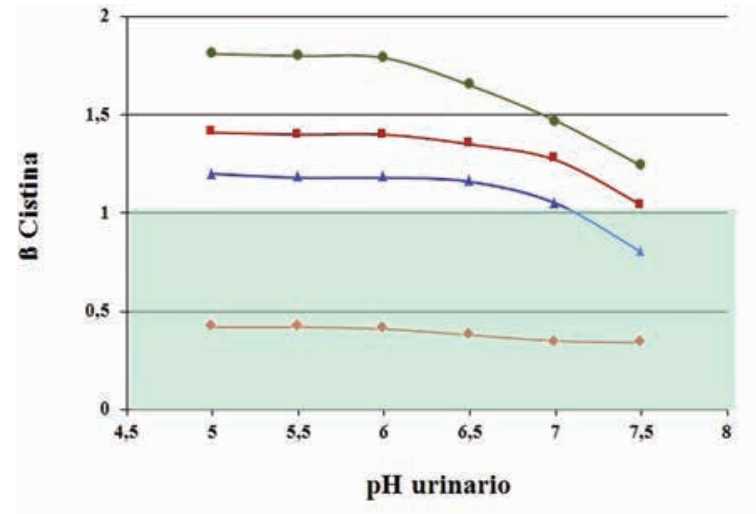

$\rightarrow-C y s=600 \mu \mathrm{mol} L \quad \rightarrow C y s=1200 \mu \mathrm{mol} / \mathrm{C} \quad \rightarrow-C y s=1500 \mu \mathrm{mol} / \mathrm{L} \quad \rightarrow-C y s=2000 \mu \mathrm{mol} / \mathrm{L}$

Fig. 3 - Effetti della variazione del pH sullo stato di saturazione urinaria per la Cistina (ßCys), calcolati per quattro differenti valori di cistinuria.

La biochimica urinaria (Tab. II) dimostrò valori di saturazione rispetto all'acido urico (ßAU) spiccatamente inferiori ai livelli critici e valori di ßCaOx ampiamente nei limiti della norma. Pertanto, nel complesso, i dati morfologici e biochimici apparivano poco tipici sia per una litiasi calcica sia per una forma urica.

Un test di Brand effettuato su un campione urinario fornito estemporaneamente dalla paziente risultò positivo, indicando con ciò la presenza di livelli di Cistina urinaria $>300 \mu \mathrm{mol} / \mathrm{L}$ (7). Conseguentemente, fu effettuato il dosaggio specifico della Cistina urinaria (Tab. III).

\section{Diagnosi}

Il dosaggio della Cistina urinaria, sia in valori assoluti sia dopo normalizzazione per la creatinina, risultò indicativo di cistinuria omozigote $(8,9)$.

L'escrezione urinaria di Cistina è normale negli eterozigoti di tipo A e aumentata nella quasi totalità di quelli di tipo $B$, con livelli comunque decisamente inferiori rispetto a quelli dei soggetti omozigoti (10).

La debole radio-opacità dei calcoli di Cistina, osservata in questo caso, è confermata dai riscontri della letteratura (11).

\section{Terapia}

In urine con valori di pH intorno a 6.5-7, la Cistina tende a precipitare quando la sua concentrazione supera il valore di $1000 \mu \mathrm{mol} / \mathrm{L}$; se il pH è inferiore, essa precipita anche a concentrazioni inferiori a $1000 \mu \mathrm{mol} / \mathrm{L}$ (Fig. 3).

In questi casi, l'obiettivo terapeutico è quello di alcalinizzare e diluire le urine quanto basta per mantenere i livelli di Cistina entro i limiti di relativa sicurezza (per esempio, $<800 \mu \mathrm{mol} / \mathrm{L}$ a pH 6.5-7).

Dal punto di vista dietetico, diete a basso contenuto di cisti- na non hanno dimostrato particolari vantaggi del ridurre la cistinuria, mentre appare più marcato l'effetto di un'alimentazione povera di sodio, che può ridurre l'escrezione urinaria di tutti gli aminoacidi, inclusa la Cistina (12).

Ove si ritenga improbabile riuscire a mantenere costantemente le concentrazioni di Cistina desiderate con la sola terapia idropinica, è necessario migliorarne la solubilizzazione mediante una terapia con Tiopronina (alfa-mercaptopropionil-glicina) (13).

Alla paziente furono fornite le seguenti prescrizioni terapeutiche:

- $\quad$ bere quanto necessario per ottenere una diuresi non inferiore a 3 litri;

- $\quad$ seguire un'alimentazione normoproteica, a basso contenuto di sodio;

- assumere Citrato tripotassico monoidrato 2 grammi 2 volte al giorno, aumentabile a 2 grammi 3 volte al giorno qualora il $\mathrm{pH}$ urinario risultasse tendenzialmente $<6.5$;

- $\quad$ assumere Tiopronina, $250 \mathrm{mg} 2 \mathrm{cp} \times 3$;

- $\quad$ sospendere il bicarbonato di sodio.

\section{Decorso clinico}

Ipotizzando di mantenere una diuresi di 3-3.5 litri e considerando un'escrezione urinaria di Cistina abitualmente intorno a $3500 \mu \mathrm{mol} / 24 \mathrm{~h}$, con la sola terapia idropinica la paziente avrebbe ottenuto una saturazione di Cistina intorno a $1000 \mu \mathrm{mol} / \mathrm{I}$ (Tab. III), un livello ancora prossimo al limite di solubilità, con rischio di formazione di calcoli.

I dati metabolici della paziente rendono comunque conto del favorevole decorso clinico precedente, quando, grazie a un'introduzione idrica abbondante e all'autoprescrizione di alcalinizzanti, ella aveva sviluppato una malattia molto meno severa rispetto a quella del fratello.

Al momento della prima valutazione, la paziente risultò portatrice di litiasi bilaterale. Le modeste dimensioni dei calcoli rendevano teoricamente possibile non solo un contenimento del loro accrescimento, ma anche la loro dissoluzione a fronte di un'adeguata sottosaturazione urinaria. L'aggiunta di Tiopronina apparve, dunque, fortemente raccomandabile (13).

La Tiopronina è un complessante della Cistina, con un rapporto di neutralizzazione di 1 a 2 (una $\mu \mathrm{mol}$ di Tiopronina solubilizza $0.5 \mu \mathrm{mol}$ di Cistina). È, dunque, possibile stimare empiricamente la quota di Cistina libera urinaria con la formula: [Cistina - (Tiopronina/2)]. Nel caso in questione, la terapia con Tiopronina consenti di ottenere una quota libera di Cistina urinaria di circa $500 \mu \mathrm{mol} / \mathrm{L}$, intorno al 50\% del valore-rischio (Tab. III).

Dopo un anno di terapia, la documentazione ecografica della volumetria dei calcoli dimostrò la presenza di un calcolo di $3 \mathrm{~mm}$ ai calici medi di destra (precedente, $6 \mathrm{~mm}$ ) e un dubbio spot caliciale sinistro di $1.5 \mathrm{~mm}$ (precedente, $5 \mathrm{~mm}$ ). 


\section{Conclusioni}

La cistinuria è una malattia determinata da mutazioni geniche recessive, caratterizzata da un alterato trasporto della Cistina e degli aminoacidi dibasici Arginina, Lisina e Ornitina a livello intestinale e renale (8).

Essa può, talora, esordire clinicamente in età avanzata e avere, come nel caso qui descritto, un decorso profondamente differente in diversi membri di una stessa famiglia.

Una corretta e tempestiva diagnosi di malattia è fondamentale, giacché la terapia può efficacemente mantenere quoad vitam una funzione renale normale, in soggetti altrimenti ad alto rischio di evoluzione verso l'uremia terminale.

\section{Caso clinico 5}

\section{Anamnesi}

Paziente nata nel 1965. Due gravidanze regolarmente condotte a termine, anamnesi patologica remota non significativa. La calcolosi renale esordì nel 1990, con coliche recidivanti ed espulsione di un calcolo non analizzato.

Seguì un periodo di benessere fino al 1995, quando vi fu l'espulsione di un secondo calcolo, risultato di Ossalato di Calcio monoidrato. Un'ecografia eseguita in Pronto Soccorso, in fase pre-espulsiva, documentò la presenza di un calcolo iuxta-vescicale sinistro, con moderata idroureteronefrosi a monte, e di qualche spot iperecogeno a livello renale bilateralmente.

Quando la paziente giunse presso il nostro Ambulatorio era asintomatica, in buone condizioni generali, non assumeva alcuna terapia farmacologica e seguiva un regime alimentare vario ed equilibrato, con un apporto idrico di circa 1.5 litri al giorno.

\section{Indagini biochimiche e strumentali}

I risultati salienti delle indagini da noi prescritte sono riportati nella Tabella IV.

In condizioni basali, a dieta libera e senza interferenze farmacologiche, l'elevato rischio litogeno urinario per l'ossalato di calcio ( $ß \mathrm{CaOx}=8.4$ ) appariva sostenuto in gran parte dagli aumentati livelli di ossaluria (confermati anche dopo normalizzazione per la creatininuria), mentre calciuria e citraturia erano del tutto normali.

\section{Diagnosi}

L'ossalato urinario è un end-product del metabolismo e, generalmente, circa il 5-10\% della quantità escreta giornalmente con le urine è direttamente riconducibile a un'ingestione alimentare di ossalato in quanto tale (14).

In caso di valori di ossaluria superiori alla norma, da valutarsi anche dopo normalizzazione rispetto alla creatininuria, sono possibili tre ipotesi diagnostiche:

a) iperossaluria secondaria a cospicua introduzione di alimenti a elevato contenuto di ossalato o di suoi precursori (glicina, acido ascorbico) $(14,15)$;

b) iperossaluria enterica (nelle malattie infiammatorie intestinali e nelle sindromi da malassorbimento, inclusi gli esiti di chirurgia bariatrica) $(16,17)$;

c) iperossaluria primitiva, geneticamente trasmissibile come carattere recessivo (18).

Nel caso in esame, non sussistevano condizioni di aumentato apporto dietetico di ossalato o di suoi precursori e non vi era evidenza di enteropatia. Conseguentemente, fu posto il sospetto di Iperossaluria Primitiva.

Per conferma, furono programmate ulteriori indagini bioumorali (dosaggio dell'ossalato plasmatico e del glicolato urinario) e di biologia molecolare (determinazione dell'attività dell'Alanina-Gliossilato Aminotransferasi epatica, AGT), i cui esiti sono riportati nella Tabella $V(19,20)$.

I livelli di ossalemia risultarono circa 10 volte superiori rispetto alla norma, confermando la diagnosi di Iperossaluria Primitiva. In particolare, gli elevati valori di escrezione urinaria di Glicolato suggerivano una forma di tipo 1 (19).

La diagnosi biochimica fu confermata dalla misurazione dell'attività enzimatica dell'AGT su tessuto bioptico epatico fresco, dove furono riscontrati valori pari al $40 \%$ della norma (20). La presenza di un discreto grado di attività residua, compatibile con la diagnosi, giustifica la moderata severità metabolica e clinica della malattia.

In epoca successiva, la paziente fu sottoposta anche a uno studio genetico, che confermò definitivamente la diagnosi documentando una mutazione in omozigosi 630G>A del gene AGXT.

\section{Terapia}

La mutazione genetica individuata è caratterizzata da una sensibilità dell'AGT epatica alla piridossina, nel senso che la Vitamina B6 ha un effetto di stimolo sull'attività enzimatica (21).

Conseguentemente, fu impostata una terapia con Piridossina 300 mg $1 \mathrm{cp}$ die, in aggiunta a provvedimenti di tipo più generale, idonei a contenere la sovrasaturazione urinaria per il calcio ossalato, come l'ingestione di almeno 2.5 litri di acqua al giorno, un'alimentazione normocalcica-ipo-ossalica e l'assunzione di citrato di potassio $(2 \mathrm{~g} \times 3)$.

\section{Decorso clinico}

Dopo 6 mesi di terapia continuativa con Piridossina e Citrato di Potassio, vi furono una riduzione dell'ossaluria di oltre il 35\% e un aumento della citraturia di oltre il $50 \%$, con una conseguente diminuzione della ßCaOx (Tab. IV, colonna Vit. B6-I).

I livelli di ossalato plasmatico e quelli di glicolato plasmati- 
TABELLA IV - Caso clinico 5. Dati metabolici

\begin{tabular}{|c|c|c|c|c|}
\hline & Val. normali & Basale & Vit. B6-I & Vit. B6-II \\
\hline Creatininemia (mg/dL) & $0.5-1.3$ & 0.7 & 0.7 & 0.8 \\
\hline Clear. Creatinina $(\mathrm{mL} / \mathrm{min})$ & $70-140$ & 76 & 81 & 74 \\
\hline Calcemia totale $(\mathrm{mg} / \mathrm{dL})$ & $8.2-10.2$ & 9.5 & 9.4 & 9.3 \\
\hline Fosforemia ( $m g / d L)$ & $2.5-4.5$ & 3.7 & 3.4 & 3.2 \\
\hline Fosfaturia (mmol/24h) & $13-52$ & 21.9 & 19.1 & 21.7 \\
\hline Calciuria (mmol/24h) & $2.5-9$ & 3.6 & 2.9 & 3.1 \\
\hline Calciuria $(m g / k g)$ & $<4$ & 2.3 & 1.8 & 2.0 \\
\hline Ossaluria (mmol/24h) & $<0.55$ & 0.69 & 0.44 & 0.38 \\
\hline Ossaluria/Creat. ( $\mathrm{mmol} / \mathrm{mmol})$ & $12-55$ & 92 & 64 & 49 \\
\hline Citraturia (mmol/24h) & $1.5-6.5$ & 2.8 & 4.2 & 5.2 \\
\hline $\mathrm{pH}$ urinario & $5-7$ & 6.42 & 6.6 & 6.9 \\
\hline Diuresi $(m L / 24 h)$ & & 1650 & 2000 & 2460 \\
\hline BCaOx & Satur. $=1$ & 8.4 & 5.2 & 3.7 \\
\hline B bsh & Satur. $=1$ & 1.1 & 0.6 & 0.8 \\
\hline
\end{tabular}

TABELLA V - Caso clinico 5. Valori di ossalato e glicolato basali e in corso di terapia con Vitamina B6

\begin{tabular}{lccc}
\hline & Val. normali & Basale & Vit. B6-I \\
\hline Ossalato sierico $(\mu \mathrm{mol} / \mathrm{L})$ & $1.2-3$ & 21 & 1.5 \\
Glicolato sierico $(\mu \mathrm{mol} / \mathrm{L})$ & $3-13$ & 36 & 11 \\
Glicolato/Creatinina urinari $(\mu \mathrm{mol} / \mathrm{mmol})$ & $13-71$ & 179 & 64 \\
\hline
\end{tabular}

co e urinario rientrarono in limiti di normalità (Tab. V, colonna Vit. B6-I), documentando l'efficacia della Piridossina sull'attività enzimatica dell'AGT.

L'aderenza alla terapia farmacologica fu scrupolosa per otto anni, durante i quali non vi furono segni clinici o morfologici di attività di malattia.

Seguì un periodo di circa tre anni di scarsissima aderenza terapeutica, culminata nell'insorgenza di un'uropatia ostruttiva acuta, causata dall'incuneamento di un calcolo di $7 \mathrm{~mm}$ nell'uretere destro. Fortunatamente, all'ecografia, non risultò la presenza di altri calcoli urinari.

L'effetto metabolico della sospensione della Piridossina fu documentato dal riscontro, su un campione urinario estemporaneo raccolto in Pronto Soccorso, di un rapporto ossalato/creatinina francamente patologico (101 $\mu \mathrm{mol} / \mathrm{mmol}$; v.n. $<55)$, con valori sostanzialmente analoghi a quelli riscontrati all'epoca della diagnosi di iperossaluria.

Il calcolo ureterale fu, poi, rimosso endoscopicamente e fu ripristinata la terapia farmacologica originaria.

Quattro anni più tardi, in condizioni di aderenza terapeuti- ca ottimale, il profilo metabolico risultò in linea con quello già riscontrato nei primi anni di terapia regolare, con una sostanziale normalizzazione dell'ossaluria e un buon controllo del rischio litogeno urinario (Tab. IV, colonna Vit. B6-II).

L'ecografia renale dimostrò una sostanziale inattività della malattia (assenza di calcoli renali, dubbi spot iperecogeni sparsi).

\section{Conclusioni}

L'iperossaluria primitiva può manifestarsi con quadri clinici e laboratoristici molto variabili. Anche se assai sporadicamente, nelle forme meno gravi essa può esordire in età adulta.

Quando, in un paziente litiasico, si riscontrino livelli di ossaluria superiori alla norma, confermati anche dopo normalizzazione per la creatininuria, in assenza di condizioni di eccessiva introduzione dietetica o di enteropatie deve essere sospettata la presenza di un'iperossaluria primitiva.

La determinazione dei livelli di glicolato urinario e dell'attività dell'AGT epatica, un tempo indispensabile per la confer- 
ma diagnostica, può, oggi, essere sostituita dalla tipizzazione genetica, in grado anche di orientare sull'eventuale sensibilità alla piridossina nelle forme di tipo 1 .

Nelle forme sensibili alla piridossina, una terapia adeguata e scrupolosa può consentire di ottenere rilevanti successi terapeutici, riducendo il rischio di recidive e di evoluzione verso l'insufficienza renale. Di qui, l'importanza di una diagnosi precoce della malattia e della sua eventuale responsività alla Vitamina B6.

\section{Disclosures}

Financial support: No financial support was received for this submission.

Conflict of interest: The authors have no conflict of interest.

\section{Bibliografia}

1. Pak $\mathrm{CY}$, Poindexter JR, Adams-Huet B, Pearle MS. Predictive value of kidney stone composition in the detection of metabolic abnormalities. Am J Med. 2003;115:26-32.

2. Gücük $A$, Uyetürk U. Usefulness of Hounsfield unit and density in the assessment and treatment of urinary stones. World J Nephrol. 2014;3(4):282-6.

3. Sakhaee K, Adams-Huet B, Moe OW, Pak CY. Pathophysiologic basis for normouricosuric uric acid nephrolithiasis. Kidney Int. 2002;62:971-9.

4. Sakhaee K, Maalouf NM, Sinnott B. Kidney Stones 2012: Pathogenesis, Diagnosis, and Management. J Clin Endocrinol Metab. 2012;97:1847-60.

5. Pak CY, Sakhaee K, Fuller C. Successful management of uric acid nephrolithiasis with potassium citrate. Kidney Int. 1986;30: 422-8.

6. Abate N, Chandalia M, Cabo-Chan AV Jr, Moe OW, Sakhaee K. The metabolic syndrome and uric acid nephrolithiasis: novel features of renal manifestation of insulin resistance. Kidney Int. 2004;65:386-92.

7. Brand E, Harris MM, Billon S. Cystinuria: excretion of a cystine complex which decomposes in the urine with the liberation of free cystine. J Biol Chem. 1930;86:315-31.

8. Chillaron J, Font-Llitjos M, Fort J, et al. Pathophysiology and treatment of cystinuria. Nat Rev Nephrol. 2010;6:424-34.

9. Andreassen KH, Pedersen KV, Osther SS, et al. How should patients with cystine stone disease be evaluated and treated in the twenty-first century? Urolithiasis. 2016;44:65-76.

10. Dello Strologo L, Pras E, Pontesilli C, et al. Comparison between SLC3A1 and SLC7A9 cystinuria patients and carriers: a need for a new classification. J Am Soc Nephrol. 2002;13(10):2547-53.

11. Patel SR, Wagner LE, Lubner MG, Nakada SY. Radiopacity and hounsfield attenuation of cystine urolithiasis: case series and review of the literature. J Endourol. 2014;28:472-5.

12. Lindell A, Denneberg T, Edholm E, Jeppsson JO. The effect of sodium intake on cystinuria with and without tiopronin treatment. Nephron. 1995;71:407-15.

13. Pak CY, Fuller C, Sakhaee K, et al. Management of cystine nephrolithiasis with $\alpha$-mercapto propionylglycine. J Urol. 1986; 136:1003-8.

14. Holmes RP, Goodman HO, Assimos DG. Contribution of dietary oxalate to urinary oxalate excretion. Kidney Int. 2001;59: 270-6.

15. Baker EM, Saari JC, Tolbert BM. Ascorbic acid metabolism in man. Am J Clin Nutr. 1966;19:317-78.

16. Parks JH, Worcester EM, O'Connor RC, Coe FL. Urine stone risk factors in nephrolithiasis patients with and without bowe disease. Kidney Int. 2003;63(1):255-65.

17. Asplin JR, Coe FL. Hyperoxaluria in kidney stone formers treated with modern bariatric surgery. J Urol 2007;177:565-9.

18. Hoppe B. An update on primary hyperoxaluria. Nat Rev Nephrol. 2012;8(8):467-75.

19. Petrarulo M, Vitale C, Facchini P, Marangella M. Biochemical approach to diagnosis and differentiation of primary hyperoxalurias: an update. J Nephrol. 1998;11(Suppl. 1):23-8.

20. Danpure CJ, Jennings PR. Peroxysomal alanine:glyoxylate aminotransferase deficiency in primary hyperoxaluria type I. FEBS Lett. 1986;201:20-4.

21. Cellini B, Montioli R, Voltattorni CB. Human liver peroxisomal alanine:glyoxylate aminotransferase: characterization of the two allelic forms and their pathogenic variants. Biochim Biophys Acta. 2011;1814(11):1577-84. 